\title{
Modeling of Plasma Formation in Rarefied Hypersonic Entry Flows
}

\author{
Iain D. Boyd* \\ University of Michigan, Ann Arbor, Michigan, 48109-2140, USA
}

\begin{abstract}
When vehicles re-enter the earth's atmosphere from space, the hypersonic conditions are sufficiently energetic to generate ionizing reactions. The production of a thin plasma layer around a hypersonic vehicle can block radio waves sent to and from the vehicle leading to communications blackout. For earth entry from orbit, the maximum energy involved in molecular collisions requires only associative ionization of air-species to be considered. In the present study, the modeling of such reactions is considered in detail using the direct simulation Monte Carlo (DSMC) method. For typical earth entry conditions, with a velocity near $8 \mathrm{~km} / \mathrm{s}$, it is shown that the average ionizing reaction probabilities are small. Special numerical techniques must therefore be used in the DSMC technique in order to numerically resolve these reactions. Additional simulation problems arise from the relatively small mass of the electrons in comparison to the other atoms and molecules in these flow fields. Artificially increasing the electron mass greatly increases computational efficiency, and the viability of this approach is investigated. Simulation results are presented for conditions corresponding to the RAM-C II hypersonic flight experiment that gathered measurements of electron number density. It is demonstrated that simulation results for electron number density in this energy regime are relatively insensitive to the mass of the electrons. Direct comparison of DSMC results with the RAM-C II measurements for electron number density show excellent agreement.
\end{abstract}

\section{Introduction}

During entry into the Earth's atmosphere of a hypersonic vehicle at sufficiently high velocity, a plasma layer is formed due to ionizing collisions within the shock heated air. For entry velocities at or below orbital speed $(7.8 \mathrm{~km} / \mathrm{s})$, the level of ionization is relatively weak and the plasma layer is sometimes referred to as a plasma sheath. Even for such a weak plasma, the well-known phenomenon of communications blackout occurs. In communications blackout, the plasma sheath surrounding a hypersonic vehicle makes it difficult to transmit radio waves to and from the vehicle thereby causing significant interference with operation of the vehicle. At higher entry velocities associated with lunar, Mars, and other capsule return, the levels of ionization can be significant playing an important role in determining the convective and radiative heat fluxes to the vehicle.

The aerothermodynamics analysis of the high altitude portions of a hypersonic entry trajectory are routinely performed using the direct simulation Monte Carlo (DSMC) method. ${ }^{1}$ For example, the DSMC technique has been applied in the past to analyze hypersonic air flows at velocities up to about $10 \mathrm{~km} / \mathrm{s}^{2,3}$ These studies included ionizing reactions using procedures for simulating the electrons in DSMC proposed by Bird. ${ }^{2}$ An attempt by Bird ${ }^{4}$ to assess these electron simulation schemes by comparing with experimental measurements made in a hypersonic flight was unsuccessful in part due to the limited computer resources available at the time.

The primary goal of this study is to develop and apply new numerical schemes to improve the ability of the DSMC method to simulate the plasma sheath formed around a hypersonic vehicle entering the atmosphere at orbital velocity under rarefied flow conditions. The simulations are required for assessment of communications blackout mitigation schemes. Using modern computers, it is now possible to assess these schemes using the same available flight measurements considered in. ${ }^{4}$

* Professor, Department of Aerospace Engineering, 1320 Beal Ave, AIAA Associate Fellow. 
Details of the flight experiment and the levels of ionization present in the associated flow field are first described. Existing methods for simulating ionization using the DSMC technique are reviewed. New numerical schemes required for appropriate resolution of the plasma are described. Results obtained through application of the new schemes to the conditions of the flight experiment are presented and final conclusions are formulated.

\section{The RAM-C II Flight Experiment}

The Radio Attenuation Measurement (RAM) experiments involved a series of hypersonic entry flights designed to study communications blackout. The vehicle of interest here (RAM-C II), consisted of a cone with a spherical nose cap of radius, $R_{N}=0.1524 \mathrm{~m}$, a cone angle of 9 deg., and a total length of about $1.3 \mathrm{~m}$. While entering at orbital velocity $(7.8 \mathrm{~km} / \mathrm{s})$, the RAM-C II experiment made measurements from about $90 \mathrm{~km}$ to $60 \mathrm{~km}$ altitude. Electron number density was measured using two different diagnostics at several locations in the plasma sheath surrounding the vehicle. ${ }^{5,6}$ A series of reflectometers was used to measure the maximum plasma density along lines normal to the vehicle surface in four different locations. A rake of Langmuir probes measured variation in the plasma density across the plasma sheath at a location $Z / R_{N}=8.1$, near the rear of the vehicle. A schematic diagram of the vehicle geometry and the instrumentation placement is shown in Fig. 1.

The RAM-C II data have been used in several previous CFD studies ${ }^{7-9}$ to assess ionization models. Bird $^{4}$ attempted to use the DSMC method to study the RAM-C II data collected at $81 \mathrm{~km}$ but obtained inconclusive results in part due to the inability to use a large enough number of particles given the computer resources of that time. Note that Bird's study was conducted more than 15 years ago. The CFD analyses of the $81 \mathrm{~km}$ RAM-C II flow indicate that the mole fraction of electrons ranges from about $10^{-3}$ and below. Resolving such a trace amount of any species with the DSMC technique requires special treatment. The present study again considers the $81 \mathrm{~km}$ RAM-C II data and seeks to apply modern DSMC methods as well as faster computers to this flow configuration. The overall goal of the work is to produce a validated DSMC approach for addressing communications blackout issues at high altitude.

\section{Simulation of Ionizing Reactions}

The presence of electrons in a DSMC computation presents a couple of difficulties due to their mass being five orders of magnitude lower than the heavy air species such as nitrogen and oxygen. First, if all species are equilibrated to the same translational temperature, due to their very low mass, the electrons diffuse at velocities that are more than two orders of magnitude higher than the air species. However, the strong electrostatic attraction between electrons and ions leads to significant limitation of the transport of the electrons. Indeed, the assumption of ambi-polar diffusion requires that electrons and ions diffuse at the same rate. First proposed by Bird, ${ }^{4}$ ambi-polar diffusion is implemented in the DSMC technique by forcing the motion of an electron to follow that of the ion with which it was first created in the simulation. This ambi-polar diffusion model is employed in the current work.

The second problem created by the very small mass of an electron concerns simulation of collisions. The collision probability in the DSMC technique is proportional to the product of the collision cross section and the relative velocity of a pair of particles. If one of the particles is an electron, then the pair will possess a very large relative velocity. Electrons therefore undergo a much higher rate of collisions in comparison to the heavy species. Thus, DSMC computations involving electrons either have to employ a time step that is significantly smaller than that needed for a simulation under the same flow conditions without electrons, or sub-cycling of collisions must be performed. In sub-cycling, the computation of collisions is broken down into several cycles for each overall simulation time step. Both of these approaches leads to a significant increase in overall computational cost in comparison to a simulation that does not involve electrons. In the results below, the possibility of alleviating this difficulty by artificially increasing the mass of the electron is investigated.

The third area of concern for the RAM-C II simulations concerns the level of ionization that is anticipated to be low. At orbital speed of $7.8 \mathrm{~km} / \mathrm{s}$, corresponding to an energy of about $9 \mathrm{eV}$, the main reactions leading to ionization in air are:

$$
N+N \rightarrow N_{2}^{+}+E^{-}
$$




$$
\begin{gathered}
O+O \rightarrow \mathrm{O}_{2}^{+}+E^{-} \\
N+O \rightarrow N O^{+}+E^{-}
\end{gathered}
$$

It should be noted that $\operatorname{Bird}^{4}$ only included the third of the three associative ionization reactions and his results generally under predicted the electron number densities measured on RAM-C II by factors between two and ten.

In the DSMC technique, the Total Collision Energy (TCE) chemistry model ${ }^{1}$ converts chemical rate coefficients expressed in modified Arrhenius form into reaction probabilities. Using the rate coefficients recommended at high temperature by Park, ${ }^{10}$ the reaction probabilities of these three reactions are plotted in Fig. 2. From this plot, it can be seen that the activation energies for all three reactions lie below $9 \mathrm{eV}$, and that the reaction probabilities are relatively low.

In preliminary DSMC calculations of the RAM-C II flow, a very small number of these ionization reactions occurred, similar to the situation encountered in Bird's original study. ${ }^{4}$ It is therefore found necessary to employ special numerical schemes in order to generate a sufficient number of charged particles (ions and electrons) in the RAM-C II flow fields. These schemes consist of the following steps:

(1) the reaction probabilities of the three associative ionization reactions are increased by a factor, $W$;

(2) whenever an ionizing reaction occurs, several realizations, $S>1$, of the post-reaction outcome are processed.

(3) whenever an ionizing reaction occurs, the charged particles created are given a relative numerical weight of $1 /(W \times S)$. The numerical weight of a particle in the DSMC technique is the number of real molecules represented by a single particle. Most DSMC computations employ the same numerical weight for all particles. However, for simulating species occurring in trace quantities, it is useful to decrease the relative numerical weight of trace species particles so that a larger number of such particles is simulated. The relative numerical weight is taken into account in computing the species number density and in determining the number of collisions experienced by these trace particles using the DSMC trace-species algorithm of Boyd. ${ }^{11}$ In this algorithm, when two particles of different numerical weights collide, the particle with the larger numerical weight is split into two. One of these particles is given the same numerical weight as the trace particle and the second split particle is given the numerical weight needed to preserve total mass. The two particles of equal numerical weight are then collided together. Following collision, the split particles are retained with the probabilities given by the ratio of their new and original numerical weights. This scheme conserves momentum and energy when averaged over many collisions. Full details of the procedures are provided in. ${ }^{11}$

In the simulation presented below, the ionizing reaction probabilities are increased by a factor of $W=30$ (to make sure that the maximum probability is still less than one) and four post-collision outcomes are processed for each single ionization event $(S=4)$. Use of these special steps therefore makes it possible to increase the number of charged particles in the DSMC computation by a factor of about 120 . This is a sufficient increase to make it possible to obtain sufficient numerical resolution of the properties of the charged species.

The associative ionization reactions are also simulated in the reverse direction. As explained in, ${ }^{3}$ the backward rates are expressed in modified Arrhenius form using the ratio of the forward rate coefficient and the equilibrium constant for each reaction. The forward and backward rate coefficients are listed in Table I.

\section{Details of the DSMC Flow Model}

The current computations employ a DSMC code developed specifically for hypersonic, ionized flow simulations. ${ }^{3}$ The flow is simulated as an 11-species $\left(\mathrm{N}_{2}, \mathrm{~N}, \mathrm{O}_{2}, \mathrm{O}, \mathrm{NO}, \mathrm{N}_{2}^{+}, \mathrm{N}^{+}, \mathrm{O}_{2}^{+}, \mathrm{O}^{+}, \mathrm{NO}^{+}, \mathrm{E}^{-}\right.$) reacting gas. Models are implemented for rotational ${ }^{12}$ and vibrational ${ }^{13}$ energy exchange. Most of the chemical reactions employ the TCE model except for the dissociation of nitrogen and oxygen. In some simulations, these reactions employ the Vibrationally Favored Dissociation (VFD) model. ${ }^{14}$ The VFD model makes it possible to bias the dissociation reaction probability in favor of molecules that possess a higher vibrational energy. In this way, the important phenomenon of vibration-dissociation coupling can be simulated. In the present work, the VFD favoring parameter $\phi$ is set to 2.0 for nitrogen dissociation and 0.5 for oxygen dissociation, as determined in prior work. ${ }^{14}$ As an illustration, Fig. 3 shows the VFD dissociation probabilities as a function of total collision energy and vibrational energy for nitrogen dissociation. Simulation of the dissociation reactions is a key element in simulating communications blackout because atoms are required to 
generate the charged particles via the associative ionization reactions. The sensitivity of the DSMC results to the dissociation modeling will be examined for the RAM-C II flow conditions.

The full list of chemical reactions and rate coefficients employed in the present study are provided in Table I. Note the omission in this mechanism of the direct ionization reactions:

$$
\begin{aligned}
& N+E^{-} \rightarrow N^{+}+2 E^{-} \\
& O+E^{-} \rightarrow O^{+}+2 E^{-}
\end{aligned}
$$

The activation energies for these reactions lie above $13 \mathrm{eV}$ that is higher than the collision energies experienced in the RAM-C II flow. The absence in the RAM-C II flows of chemical reactions driven by electrons suggests that it may be possible to simplify the simulation of the electrons by artificially increasing their mass. This idea is tested out in the following section.

\section{Results}

DSMC computation of the flow around the RAM-C II vehicle is performed at a flow velocity of $7.8 \mathrm{~km} / \mathrm{s}$ and flow conditions corresponding to $81 \mathrm{~km}$ altitude in the atmosphere. This is a point on the RAM-C II trajectory where measurements of electron number density are reported in. ${ }^{5,6}$ At $81 \mathrm{~km}$, the mean free path is about $0.005 \mathrm{~m}$ giving an overall Knudsen number for the flow of about 0.03 based on the spherical cap radius of RAM-C II. The analyses conducted in this study assume axial symmetry as the vehicle had a very small angle of attack. The vehicle surface is assumed to be isothermal and diffuse at a temperature of $1,500 \mathrm{~K}$, is non-catalytic to atoms, but fully catalytic to ions and electrons.

As discussed above, the presence of electrons in the flow field creates problems for the DSMC technique. The particular problem related to the need to either decrease the time step or perform collision sub-cycling greatly increases the numerical cost of the DSMC computations. It is therefore of interest to see if the cost of DSMC computations of these hypersonic flow conditions can be reduced by artificially increasing the mass of the electrons. This approach is certainly not valid when electrons drive any of the chemistry. However, as indicated in the discussions above, earth entry at orbital speed is not sufficiently energetic to produce any direct ionization by electrons.

An initial test simulation is therefore employed on a subset of the entire RAM-C II flow field representing the first $70 \mathrm{deg}$. of the spherical cap of the cone, approximately midway between the locations of the first two reflectometers (I1 and I2) indicated in Fig. 1. This region includes the stagnation streamline where the temperatures are largest and where much of the air chemistry occurs. A mesh is generated of 179 by 109 cells where the latter direction goes around the body. The mesh is adapted such that all cells are smaller than the local mean free path. Two simulations are performed:

(1) the real electron mass is employed and the collision routine is sub-cycled 30 times each iteration; and

(2) the electron mass is increased by a factor of 1,000 and no sub-cycling is employed.

The TCE model is employed for all chemical reactions including dissociation. The simulations employ about 350,000 particles at steady state and are performed for 50,000 iterations.

A general impression of the flow field can be seen in Fig. 4 where contours of translational temperature are shown. The contours are plotted at intervals of 2,000 K. These results are obtained from the simulation employing the real electron mass. A more quantitative understanding of the flow field is provided in Figs. 5 and 6 in which profiles along the stagnation streamline of various properties are shown. In Fig. 5, the mole fractions of the major species are shown. In these plots, the freestream is on the left and the vehicle surface is on the rights. The profiles illustrate the strong degree of chemical nonequilibrium present in these flows, with initial dissociation of molecular nitrogen and oxygen through the shock with partial recombination close to the vehicle surface. Figure 6 shows profiles of temperature of the translational, rotational, and vibrational modes indicating a strong degree of thermal nonequilibrium.

The effect of artificially increasing the electron mass by a factor of 1,000 is assessed in Figs. 7 and 8 . In Fig. 7, the translational temperatures for the overall gas and for the electrons are shown. The profiles obtained for the two different simulations are compared and show almost perfect agreement for the overall gas translational temperature. For the temperature of the electrons, the simulation employing the increased electron mass shows that some of the electrons are transported through the shock wave without under-going a sufficient number of collisions. Qualitatively, this phenomenon is expected since the increased electron mass must lead to a reduced collision rate of the electrons. The key question is whether this behavior leads 
to any significant differences in the electron number density, that is the key parameter for understanding communications blackout phenomena. This question is answered by Fig. 8 that shows the profiles along the stagnation streamline of the number densities of several species. Again, the profiles obtained from the two different simulations are compared and it is clear that very good agreement is obtained for all species shown. Significantly, the profiles of electron number density from the two simulations are in excellent agreement. In the front of the shock, the fact that the electron densities are so similar while the electron temperatures are so different is explained by the use of the ambi-polar diffusion assumption. Since the electrons are constrained to diffuse with the ions, the relatively poor simulation of their temperature with the increased electron mass does not significantly affect the overall transport of the electrons in the flow field.

The variation in the number densities of molecular nitrogen, the electrons and the major ion species along the stagnation streamline is shown in Fig. 9. The profiles indicate that the mole fraction of electrons is in the range of 0.01 to $0.1 \%$. The ion profiles indicate that while $\mathrm{NO}^{+}$has the highest ion concentration, all three molecular ions contribute to the total plasma density.

The profiles of maximum electron number density along lines normal to the vehicle surface are shown in Fig. 10 along with the single RAM-C II reflectometer data point. Clearly, good agreement is obtained between the two simulations and with the flight measurement.

The simulation performed with the increased electron mass requires a factor of about 10 less time to run on a computer in comparison to the case with the actual electron mass. In the latter case, the collision routine is called 30 times for every iteration. The collision routine accounts for about $30 \%$ of the cost of a single iteration without sub-cycling thus reducing the overall overhead of sub-cycling. In conclusion, the results of the small-scale, nose cap simulations clearly indicate that accurate prediction of electron number density is possible under these flow conditions through artificially increasing the electron mass by a factor of 1,000. The increased electron mass does lead to inaccuracies in simulation of the electron temperature, but that is relatively unimportant in assessment of communications blackout issues. The factor of ten increase in computational efficiency provided by this approach is of great benefit in performing the DSMC computations about the full RAM-C II vehicle.

The computational domain for the full-scale simulation is shown in Fig. 1 and employs a mesh that consists of 179 (towards the body) by 864 (along the body) cells. Once again, the mesh is adapted to ensure that each cell is smaller than the local mean free path. Each simulation employs the electron mass artificially increased by a factor of 1,000. The simulations employ more than three million particles and are run for 150,000 iterations. Results are presented below for both the TCE and VFD chemistry models for the dissociation reactions. All other reactions employ the TCE model.

The overall flow field contours of translational temperature are shown in Fig. 11. The contours reveal both the strong compression in front of the vehicle and the subsequent expansion of the flow around the shoulder of the spherical nose cap. Contours of the electron number density are shown in Fig. 12. There is a more than two orders of magnitude reduction in electron number density as a streamline is followed from the stagnation region around the side of the vehicle. This behavior is expected due to a combination of the gas expansion process but also due to the weaker compression across the outer edges of the bow shock wave. Some statistical scatter is evident in the low density contours due to relatively small numbers of electron particles that are created even when using the special numerical techniques discussed earlier.

The variation of the maximum electron number density evaluated along lines normal to the vehicle surface is shown in Fig. 13. Simulation profiles obtained with both the TCE and VFD chemistry models are compared with the RAM-C II flight data. Some sensitivity of the predicted profiles of electron number density to the dissociation model is apparent. The electron number density predicted with the VFD model is consistently about a factor of two lower than that obtained with the TCE model. The VFD model simulates a decreased dissociation rate when the vibrational temperature lies below the translational temperature, that is the case in compressed hypersonic flows. This phenomenon is illustrated in Fig. 14 that shows the profiles of nitrogen and oxygen atoms along the stagnation streamline computed using the TCE and VFD models for the dissociation reactions. The concentrations of both nitrogen and oxygen atoms predicted by the VFD model are again about a factor of two lower than that obtained with the TCE model. This reduction in atomic concentrations leads directly to a reduction in the production of electrons through the associative ionization reactions. No error bars for the RAM-C II reflectometer data are provided in ${ }^{5}$ but it is reasonable to conclude that both DSMC profiles provide very good agreement with the measurements.

Figure 15 shows comparisons between the RAM-C II flight measurements and the two different DSMC computations for the profile of electron number density across the plasma sheath at the downstream end of 
the vehicle. The RAM-C II data in this case were obtained with a rake of Langmuir probes. ${ }^{6}$ As reported in, ${ }^{6}$ the RAM-C II vehicle experienced a coning motion during re-entry with a rate of about three revolutions per second that gave rise to variation in the electron number density measured by the Langmuir probes. The error bars included in Fig. 15 for the measured data represent the variations experienced for these probes at $81 \mathrm{~km}$ for one complete revolution. The DSMC results again demonstrate sensitivity of the predicted electron number density to the two different dissociation models with the VFD model predicting an electron number density that is about a factor of two lower than the TCE model. Given the difficulties and uncertainties in making measurements in hypersonic flight conditions, the level of agreement for either DSMC result with the RAM-C II data can be considered very good.

\section{Conclusions}

In this investigation, the direct simulation Monte Carlo method was applied to analyze the hypersonic flow around the RAM-C II flight experiment under high-altitude, rarefied conditions. The primary goal of the study was to predict the electron number density field around the vehicle in order to analyze communications blackout issues.

Application of the DSMC method to ionizing flows presented several difficulties that were addressed in the present study. As in prior work, the ambi-polar diffusion assumption was invoked making an electron move around the flow field with the ion with which it was first formed. Low concentrations of the charged species made numerical resolution of their properties difficult. This problem was addressed by increasing the probability of ionizing reactions and sampling several post-ionization outcomes for each reaction. These techniques were made possible through the use of lower relative numerical weights for the charged species. The third problem encountered involved the significant increase in computational cost associated with the increased collision rates experienced by electrons due to their very small mass. Numerical experiments on a reduced size flow field demonstrated that the variation of electron number density was insensitive to artificially increasing the electron mass by a factor of 1,000. The profiles of electron temperature through the shock wave were influenced by the increased electron mass, but this is not a significant effect in terms of assessing communications blackout issues. The simulation employing the increased mass electrons was an order of magnitude faster than that employing the real electron mass.

Full simulation of the RAM-C II vehicle was performed using two different dissociation models with the electron mass increased by a factor of 1,000. The predictions of electron number density were found to be sensitive to the dissociation model employed due to the fact that electron production proceeds via associative ionization reactions in which all reactants are atoms. Comparison of the DSMC results with two different sets of RAM-C II electron number density measurements revealed excellent agreement. These are, to our knowledge, the first successful DSMC computations of the electron number densities measured by the RAM-C II flight experiment.

\section{Acknowledgments}

This work is sponsored by the Stanford Center for Turbulence Research and the Space Vehicle Technology Institute, under NASA grant NCC3-989 with joint sponsorship from the Department of Defense.

\section{References}

${ }^{1}$ Bird, G. A., Molecular Gas Dynamics and the Direct Simulation of Gas Flows Oxford University Press, Oxford, 1994.

${ }^{2}$ Bird, G. A., "Nonequilibrium Radiation During Re-entry at 10 km/s," AIAA Paper 87-1543, June 1987.

${ }^{3}$ Boyd, I. D. and Gokcen, T., "Computation of Axisymmetric and Ionized Hypersonic Flows Using Particle and Continuum Method," AIAA Journal, Vol. 32, 1994, pp. 1828-1837.

${ }^{4}$ Bird, G. A., "Computation of Electron Density in High Altitude Re-entry Flows," AIAA Paper 89-1882, June 1989.

${ }^{5}$ Grantham, W. L., "Flight Results of a 25000 Foot Per Second Reentry Experiment Using Microwave Reflectometers to Measure Plasma Electron Density and Standoff Distance," NASA Technical Note D-6062, 1970.

${ }^{6}$ Linwood-Jones, W. and Cross, A. E., "Electrostatic Probe Measurements of Plasma Parameters for Two Reentry Flight Experiments at 25000 Feet Per Second," NASA Technical Note D-6617, 1972.

${ }^{7}$ Candler, G. V. and MacCormack, R. W., "Computation of Weakly Ionized Hypersonic Flows in Thermochemical Nonequilibrium," Journal of Thermophysics and Heat Transfer, Vol. 5, 1991, pp. 266-273.

${ }^{8}$ Josyula, E. and Bailey, W., "Governing Equations for Weakly Ionized Plasma Flowfields of Aerospace Vehicles," Journal of Spacecraft and Rockets, Vol. 40, 2003, pp. 845-857. 


\footnotetext{
${ }^{9}$ Scalabrin, L. C. and Boyd, I. D., "Numerical Simulation of Weakly Ionized Hypersonic Flow for Reentry Configurations," AIAA Paper 2006-3773, June 2006.

${ }^{10}$ Park, C., Nonequilibrium Hypersonic Aerothermodynamics Wiley, New York, 1990.

${ }^{11}$ Boyd, I. D., "Conservative Species Weighting Scheme for the Direct Simulation Monte Carlo Method," Journal of Thermophysics and Heat Transfer, Vol. 10, 1996, pp. 579-585.

${ }^{12}$ Boyd, I. D., "Analysis of Rotational Nonequilibrium in Standing Shock Waves of Nitrogen," AIAA Journal Vol. 28, 1990, pp. 1997-1999.

${ }^{13}$ Boyd, I. D., "Analysis of Vibrational-Translational Energy Transfer Using the Direct Simulation Monte Carlo Method," Physics of Fluids A, Vol. 3, 1991, pp. 1785-1791.

${ }^{14}$ Haas, B. L. and Boyd, I. D., "Models for Direct Monte Carlo Simulation of Coupled Vibration-Dissociation," Physics of Fluids A, Vol. 5, 1993, pp. 478-489.
}

Table 1. Reaction rate coefficients $\left(\mathrm{m}^{3} /\right.$ molecule/s) for all reactions.

\begin{tabular}{|c|c|c|}
\hline Number & Reaction & Rate Coefficient \\
\hline $1 \mathrm{a}$ & $\mathrm{N}_{2}+\mathrm{M} \rightarrow \mathrm{N}+\mathrm{N}+\mathrm{M}$ & $1.162 \times 10^{-9} \mathrm{~T}^{-1.6} \exp (-113,200 / \mathrm{T})$ \\
\hline $1 \mathrm{~b}$ & $\mathrm{~N}_{2}+\mathrm{A} \rightarrow \mathrm{N}+\mathrm{N}+\mathrm{A}$ & $4.980 \times 10^{-8} \mathrm{~T}^{-1.6} \exp (-113,200 / \mathrm{T})$ \\
\hline $2 \mathrm{a}$ & $\mathrm{O}_{2}+\mathrm{M} \rightarrow \mathrm{O}+\mathrm{O}+\mathrm{M}$ & $3.321 \times 10^{-9} \mathrm{~T}^{-1.5} \exp (-59,400 / \mathrm{T})$ \\
\hline $2 b$ & $\mathrm{O}_{2}+\mathrm{A} \rightarrow \mathrm{O}+\mathrm{O}+\mathrm{A}$ & $1.660 \times 10^{-8} \mathrm{~T}^{-1.5} \exp (-59,400 / \mathrm{T})$ \\
\hline $3 a$ & $\mathrm{NO}+\mathrm{M} \rightarrow \mathrm{N}+\mathrm{O}+\mathrm{M}$ & $8.302 \times 10^{-15} \exp (-75,500 / \mathrm{T})$ \\
\hline $3 b$ & $\mathrm{NO}+\mathrm{A} \rightarrow \mathrm{N}+\mathrm{O}+\mathrm{A}$ & $1.826 \times 10^{-13} \exp (-75,500 / \mathrm{T})$ \\
\hline $4 \mathrm{a}$ & $\mathrm{O}+\mathrm{NO} \rightarrow \mathrm{N}+\mathrm{O}_{2}$ & $1.389 \times 10^{-17} \exp (-19,700 / \mathrm{T})$ \\
\hline $4 \mathrm{~b}$ & $\mathrm{~N}+\mathrm{O}_{2} \rightarrow \mathrm{O}+\mathrm{NO}$ & $4.601 \times 10^{-15} \mathrm{~T}^{-0.546}$ \\
\hline $5 \mathrm{a}$ & $\mathrm{O}+\mathrm{N}_{2} \rightarrow \mathrm{N}+\mathrm{NO}$ & $1.069 \times 10^{-12} \mathrm{~T}^{-1.000} \exp (-37,500 / \mathrm{T})$ \\
\hline $5 \mathrm{~b}$ & $\mathrm{~N}+\mathrm{NO} \rightarrow \mathrm{O}+\mathrm{N}_{2}$ & $4.059 \times 10^{-12} \mathrm{~T}^{-1.359}$ \\
\hline $6 a$ & $\mathrm{~N}+\mathrm{N} \rightarrow \mathrm{N}_{2}^{+}+\mathrm{E}^{-}$ & $3.387 \times 10^{-17} \exp (-67,700 / \mathrm{T})$ \\
\hline $6 \mathrm{~b}$ & $\mathrm{~N}_{2}^{+}+\mathrm{E}^{-} \rightarrow \mathrm{N}+\mathrm{N}$ & $7.274 \times 10^{-12} \mathrm{~T}^{-0.650}$ \\
\hline $7 \mathrm{a}$ & $\mathrm{O}+\mathrm{O} \rightarrow \mathrm{O}_{2}^{+}+\mathrm{E}^{-}$ & $1.859 \times 10^{-17} \exp (-81,200 / \mathrm{T})$ \\
\hline $7 \mathrm{~b}$ & $\mathrm{O}_{2}^{+}+\mathrm{E}^{-} \rightarrow \mathrm{O}+\mathrm{O}$ & $1.453 \times 10^{-4} \mathrm{~T}^{-2.412}$ \\
\hline $8 \mathrm{a}$ & $\mathrm{N}+\mathrm{O} \rightarrow \mathrm{NO}^{+}+\mathrm{E}^{-}$ & $8.766 \times 10^{-18} \exp (-32,000 / \mathrm{T})$ \\
\hline $8 b$ & $\mathrm{NO}^{+}+\mathrm{E}^{-} \rightarrow \mathrm{N}+\mathrm{O}$ & $1.321 \times 10^{-9} \mathrm{~T}^{-1.187}$ \\
\hline $9 \mathrm{a}$ & $\mathrm{O}+\mathrm{O}_{2}^{+} \rightarrow \mathrm{O}_{2}+\mathrm{O}^{+}$ & $6.641 \times 10^{-18} \mathrm{~T}^{-0.09} \exp (-18,600 / \mathrm{T})$ \\
\hline $9 b$ & $\mathrm{O}_{2}+\mathrm{O}^{+} \rightarrow \mathrm{O}+\mathrm{O}_{2}^{+}$ & $4.993 \times 10^{-18} \mathrm{~T}^{-0.004}$ \\
\hline $10 \mathrm{a}$ & $\mathrm{N}_{2}+\mathrm{N}^{+} \rightarrow \mathrm{N}+\mathrm{N}_{2}^{+}$ & $1.660 \times 10^{-18} \mathrm{~T}^{0.500} \exp (-12,100 / \mathrm{T})$ \\
\hline $10 \mathrm{~b}$ & $\mathrm{~N}+\mathrm{N}_{2}^{+} \rightarrow \mathrm{N}_{2}+\mathrm{N}^{+}$ & $2.343 \times 10^{-14} \mathrm{~T}^{-0.610}$ \\
\hline $11 \mathrm{a}$ & $\mathrm{N}_{2}+\mathrm{O}^{+} \rightarrow \mathrm{O}+\mathrm{N}_{2}^{+}$ & $1.511 \times 10^{-18} \mathrm{~T}^{0.360} \exp (-22,200 / \mathrm{T})$ \\
\hline $11 b$ & $\mathrm{O}+\mathrm{N}_{2}^{+} \rightarrow \mathrm{N}_{2}+\mathrm{O}^{+}$ & $1.978 \times 10^{-18} \mathrm{~T}^{0.109}$ \\
\hline $12 \mathrm{a}$ & $\mathrm{N}+\mathrm{NO}^{+} \rightarrow \mathrm{N}_{2}+\mathrm{O}^{+}$ & $5.670 \times 10^{-17} \mathrm{~T}^{-1.080} \exp (-12,800 / \mathrm{T})$ \\
\hline $12 \mathrm{~b}$ & $\mathrm{~N}_{2}+\mathrm{O}^{+} \rightarrow \mathrm{N}+\mathrm{NO}^{+}$ & $3.970 \times 10^{-18} \mathrm{~T}^{-0.710}$ \\
\hline $13 \mathrm{a}$ & $\mathrm{O}_{2}+\mathrm{NO}^{+} \rightarrow \mathrm{NO}+\mathrm{O}_{2}^{+}$ & $3.985 \times 10^{-17} \mathrm{~T}^{0.410} \exp (-32,400 / \mathrm{T})$ \\
\hline $13 \mathrm{~b}$ & $\mathrm{NO}+\mathrm{O}_{2}^{+} \rightarrow \mathrm{O}_{2}+\mathrm{NO}^{+}$ & $6.195 \times 10^{-16} \mathrm{~T}^{-0.050}$ \\
\hline $14 \mathrm{a}$ & $\mathrm{N}+\mathrm{NO}^{+} \rightarrow \mathrm{O}+\mathrm{N}_{2}^{+}$ & $1.195 \times 10^{-16} \exp (-35,500 / \mathrm{T})$ \\
\hline $14 \mathrm{~b}$ & $\mathrm{O}+\mathrm{N}_{2}^{+} \rightarrow \mathrm{N}+\mathrm{NO}^{+}$ & $1.744 \times 10^{-18} \mathrm{~T}^{0.302}$ \\
\hline $15 \mathrm{a}$ & $\mathrm{N}_{2}+\mathrm{O}_{2}^{+} \rightarrow \mathrm{O}_{2}+\mathrm{N}_{2}^{+}$ & $1.644 \times 10^{-17} \exp (-40,700 / \mathrm{T})$ \\
\hline $15 \mathrm{~b}$ & $\mathrm{O}_{2}+\mathrm{N}_{2}^{+} \rightarrow \mathrm{N}_{2}+\mathrm{O}_{2}^{+}$ & $4.589 \times 10^{-18} \mathrm{~T}^{-0.037}$ \\
\hline $16 \mathrm{a}$ & $\mathrm{O}+\mathrm{NO}^{+} \rightarrow \mathrm{N}+\mathrm{O}_{2}^{+}$ & $1.195 \times 10^{-17} \mathrm{~T}^{0.290} \exp (-48,600 / \mathrm{T})$ \\
\hline $16 \mathrm{~b}$ & $\mathrm{~N}+\mathrm{O}_{2}^{+} \rightarrow \mathrm{O}+\mathrm{NO}^{+}$ & $8.918 \times 10^{-13} \mathrm{~T}^{-0.969}$ \\
\hline $17 \mathrm{a}$ & $\mathrm{NO}+\mathrm{O}^{+} \rightarrow \mathrm{O}_{2}+\mathrm{N}^{+}$ & $2.324 \times 10^{-25} \mathrm{~T}^{1.900} \exp (-26,600 / \mathrm{T})$ \\
\hline $17 \mathrm{~b}$ & $\mathrm{O}_{2}+\mathrm{N}^{+} \rightarrow \mathrm{NO}+\mathrm{O}^{+}$ & $2.443 \times 10^{-26} \mathrm{~T}^{2.102}$ \\
\hline
\end{tabular}




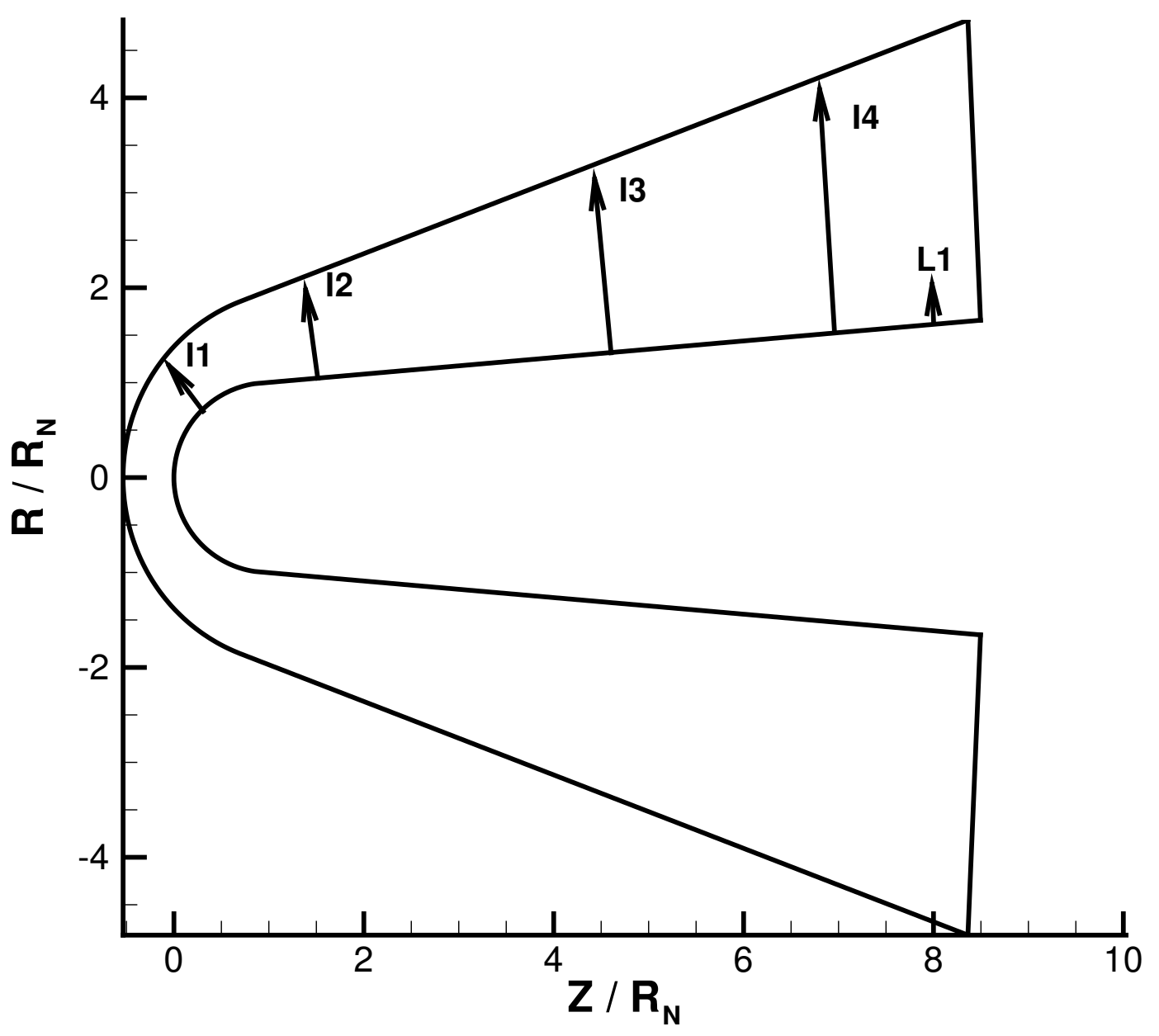

Figure 1. Computational domain for flow around the RAM-C II vehicle including the locations of the reflectometers (I1-I4) and the rake of Langmuir probes (L1). 


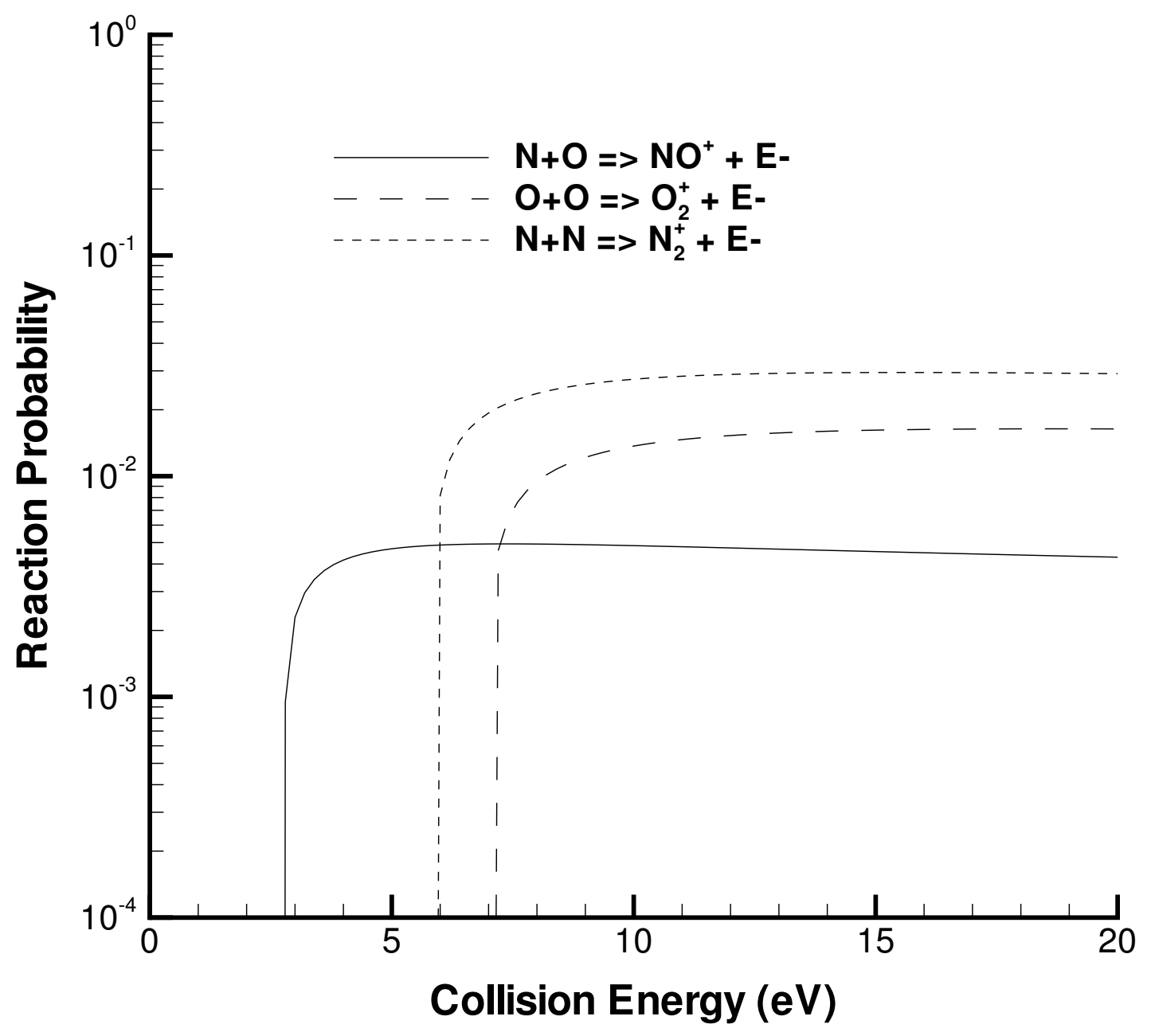

Figure 2. Probability of associative ionization obtained with the TCE model. 


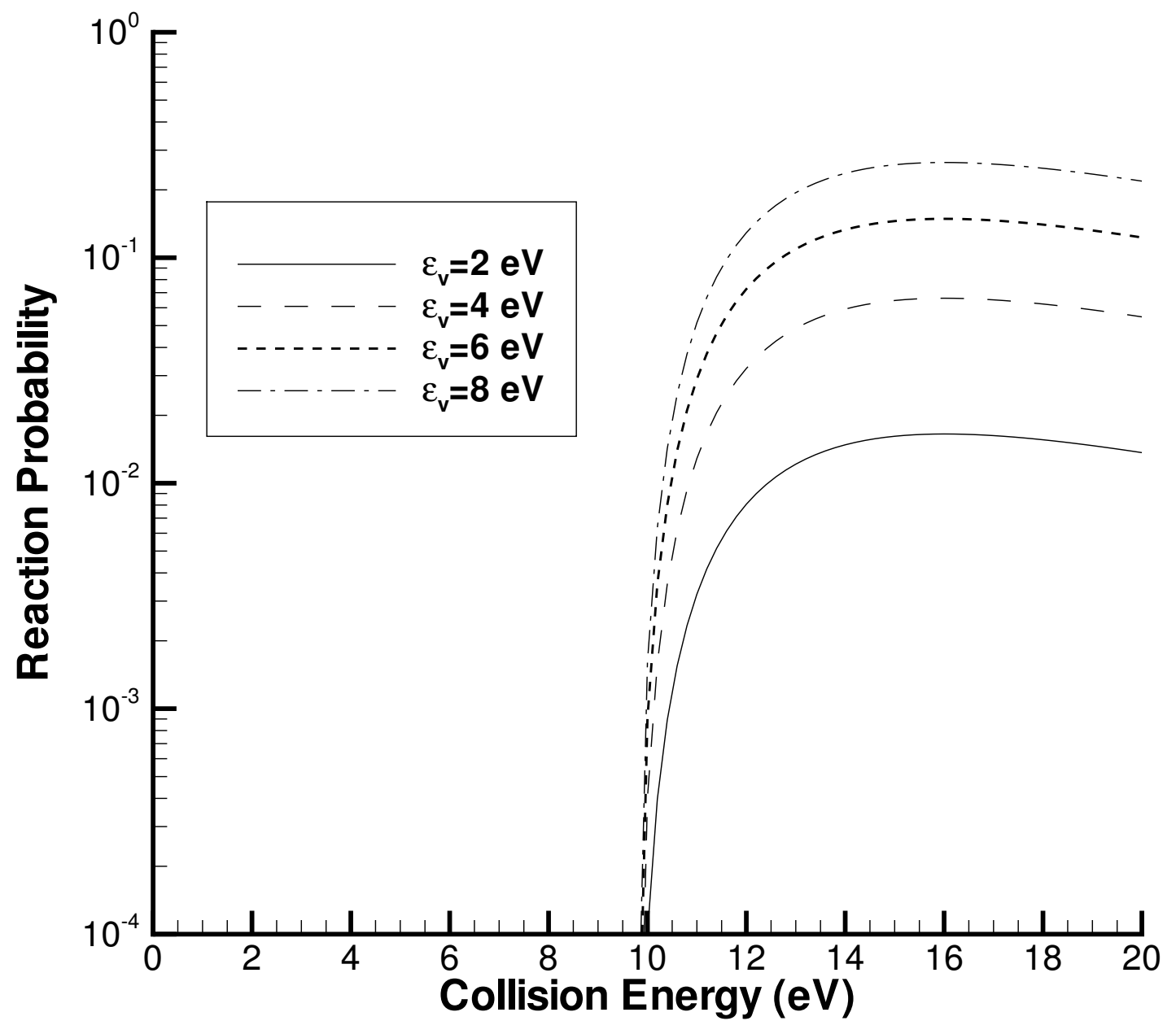

Figure 3. Probability of dissociation obtained with the VFD model for nitrogen dissociation. 


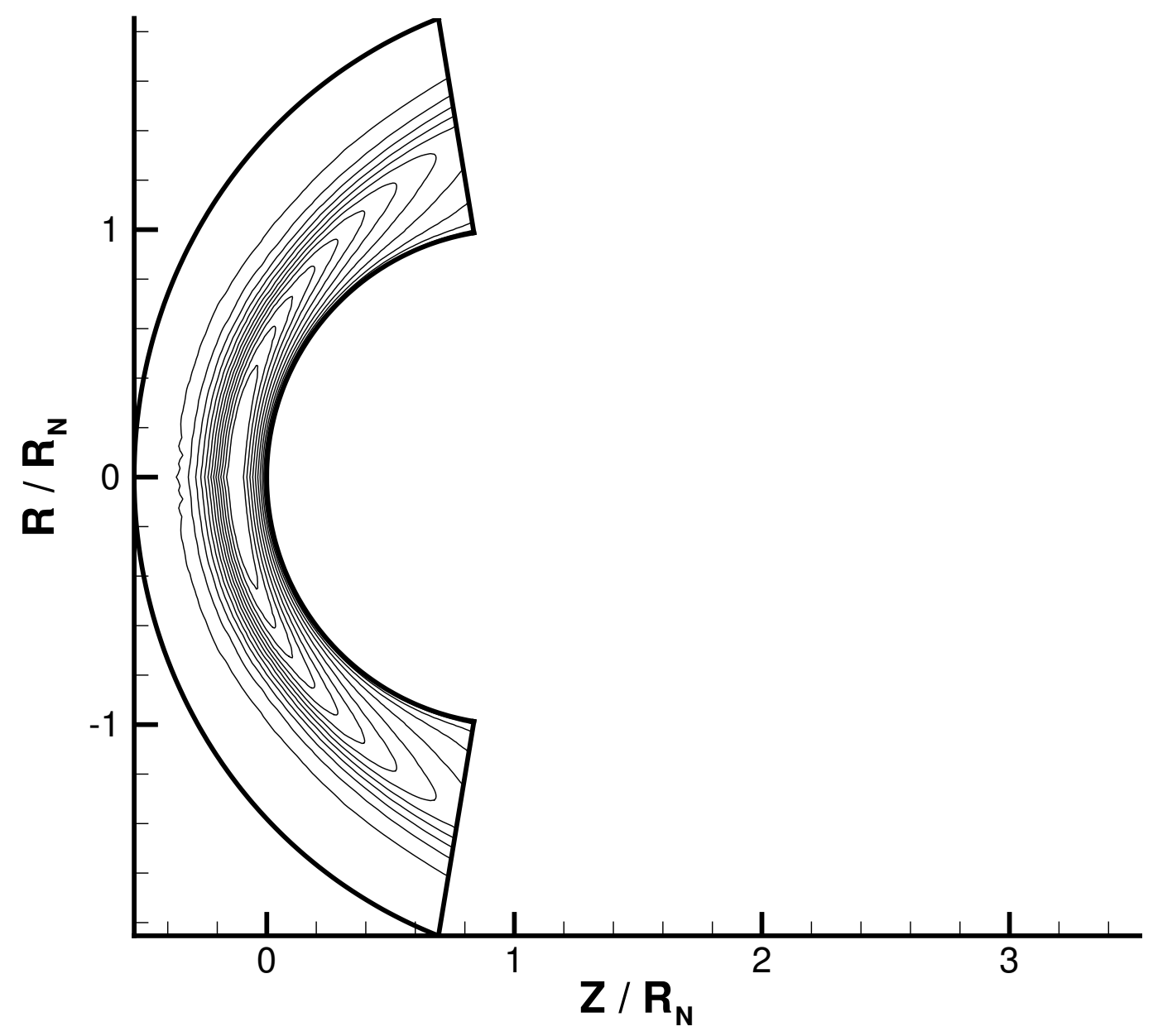

Figure 4. Temperature contours (K) for the RAM-C II nose cap region simulation. 


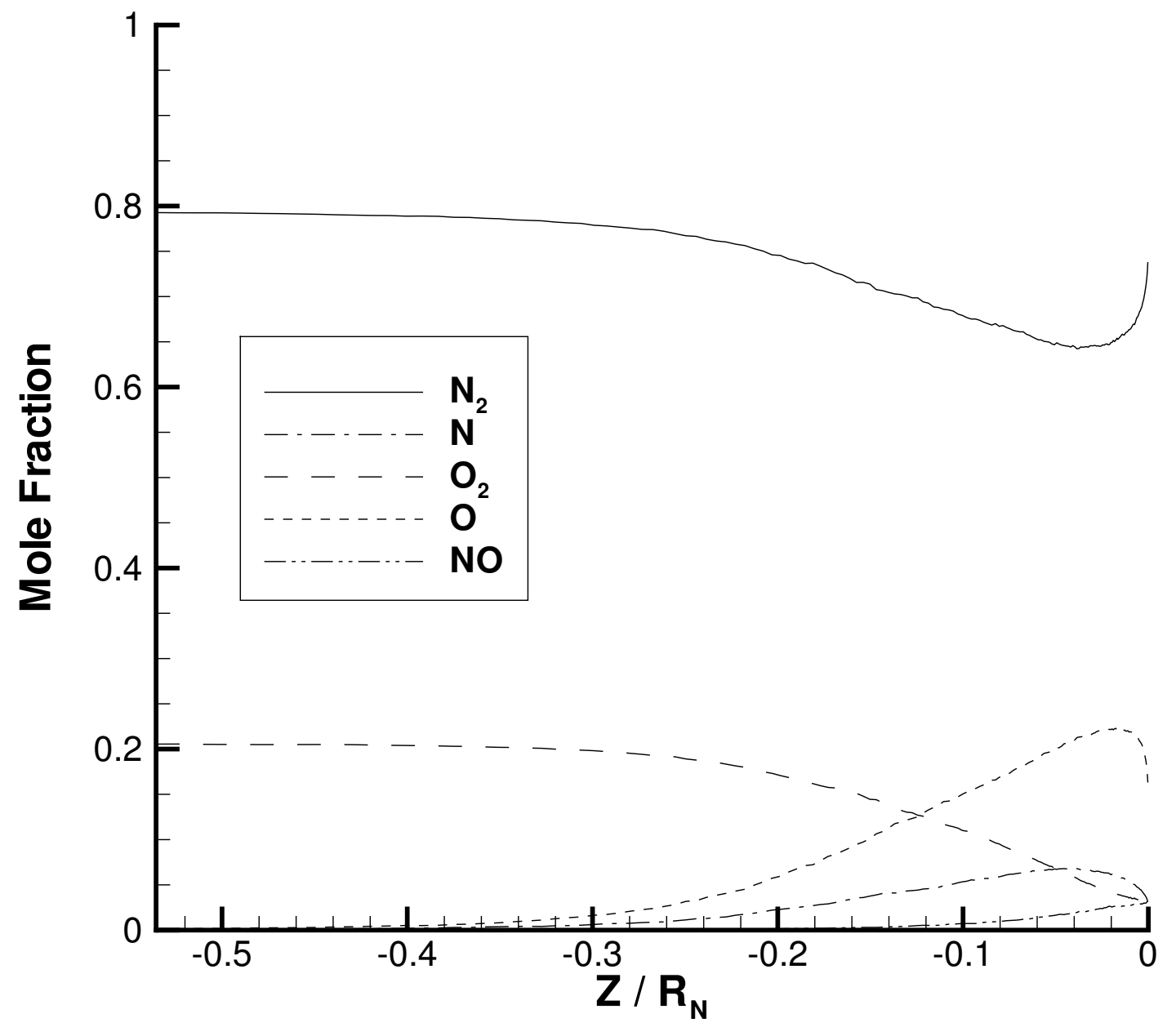

Figure 5. Mole fraction profiles along the stagnation streamline for the RAM-C II nose cap region simulation. 


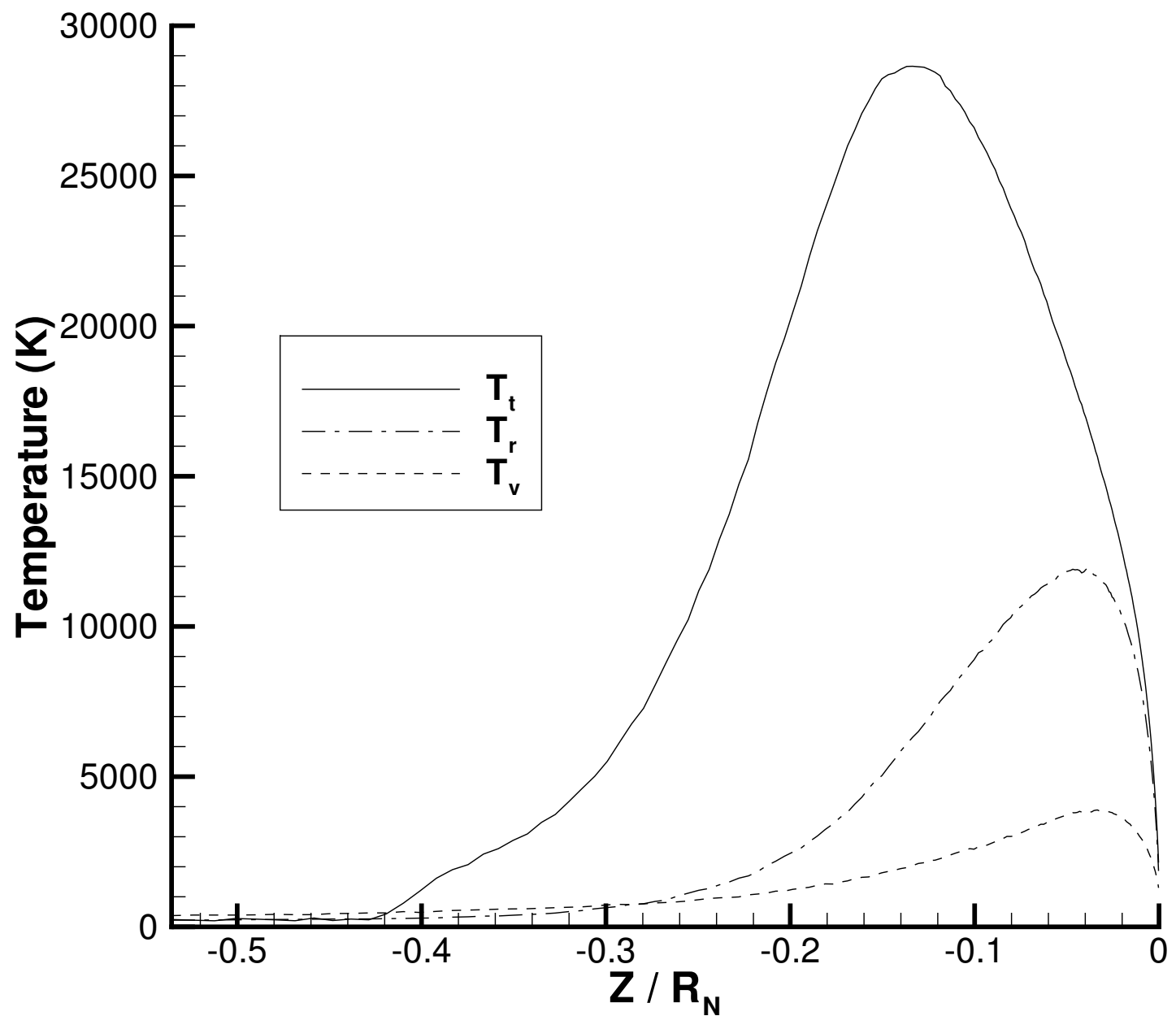

Figure 6. Temperature profiles along the stagnation streamline for the RAM-C II nose cap region simulation. 


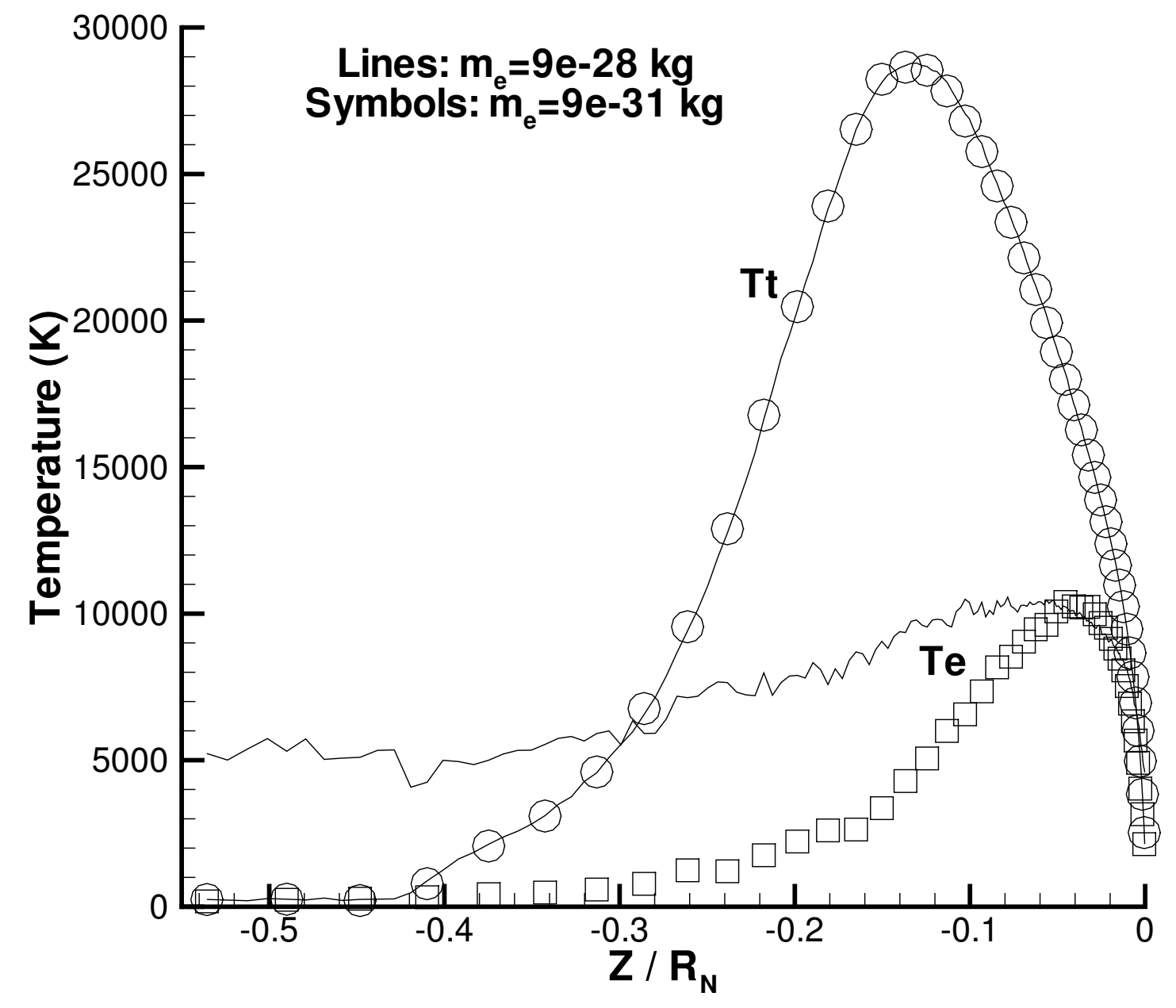

Figure 7. Temperature profiles along the stagnation streamline for the RAM-C II nose cap region simulation. 


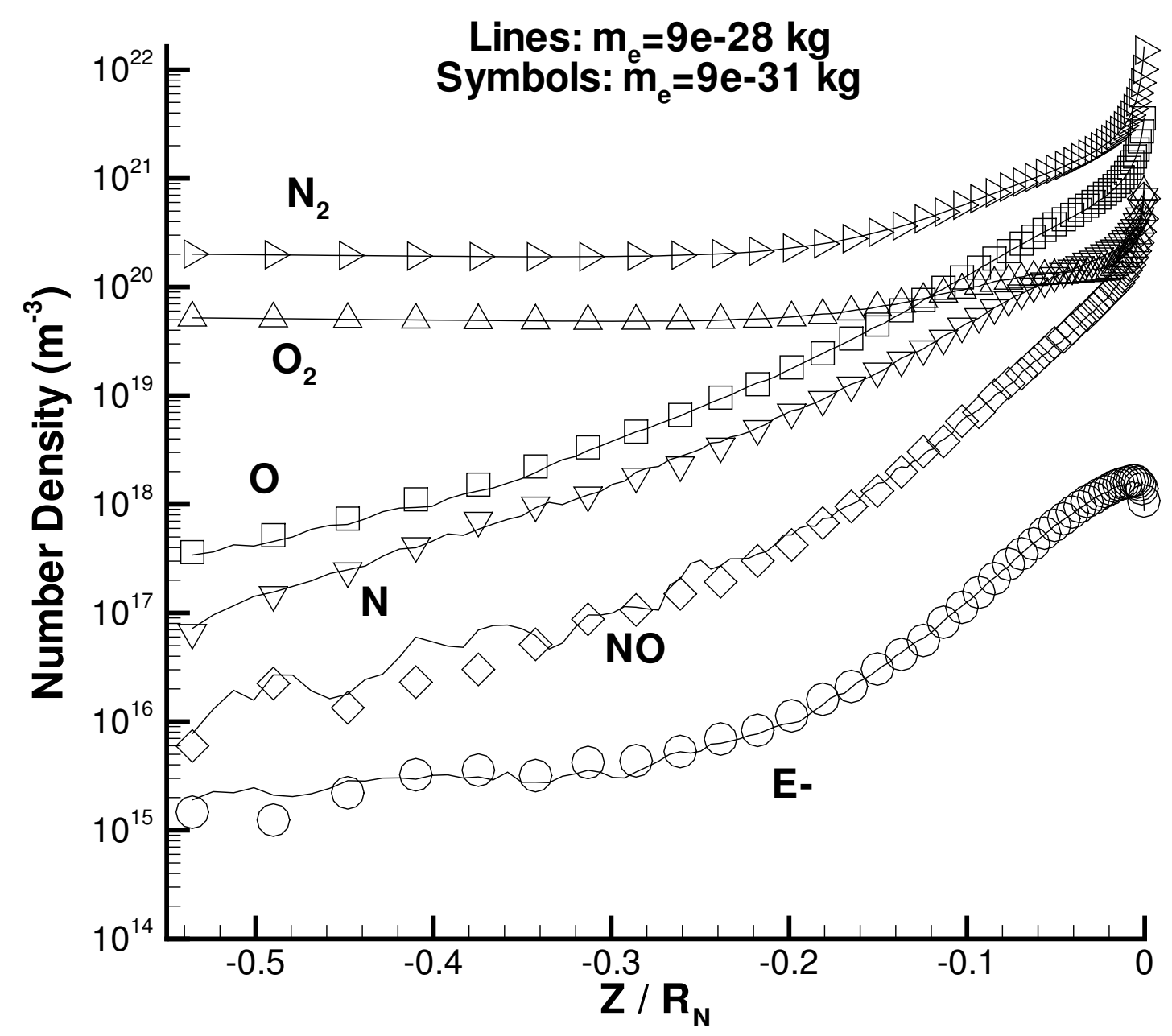

Figure 8. Species number density profiles along the stagnation streamline for the RAM-C II nose cap region simulation. 


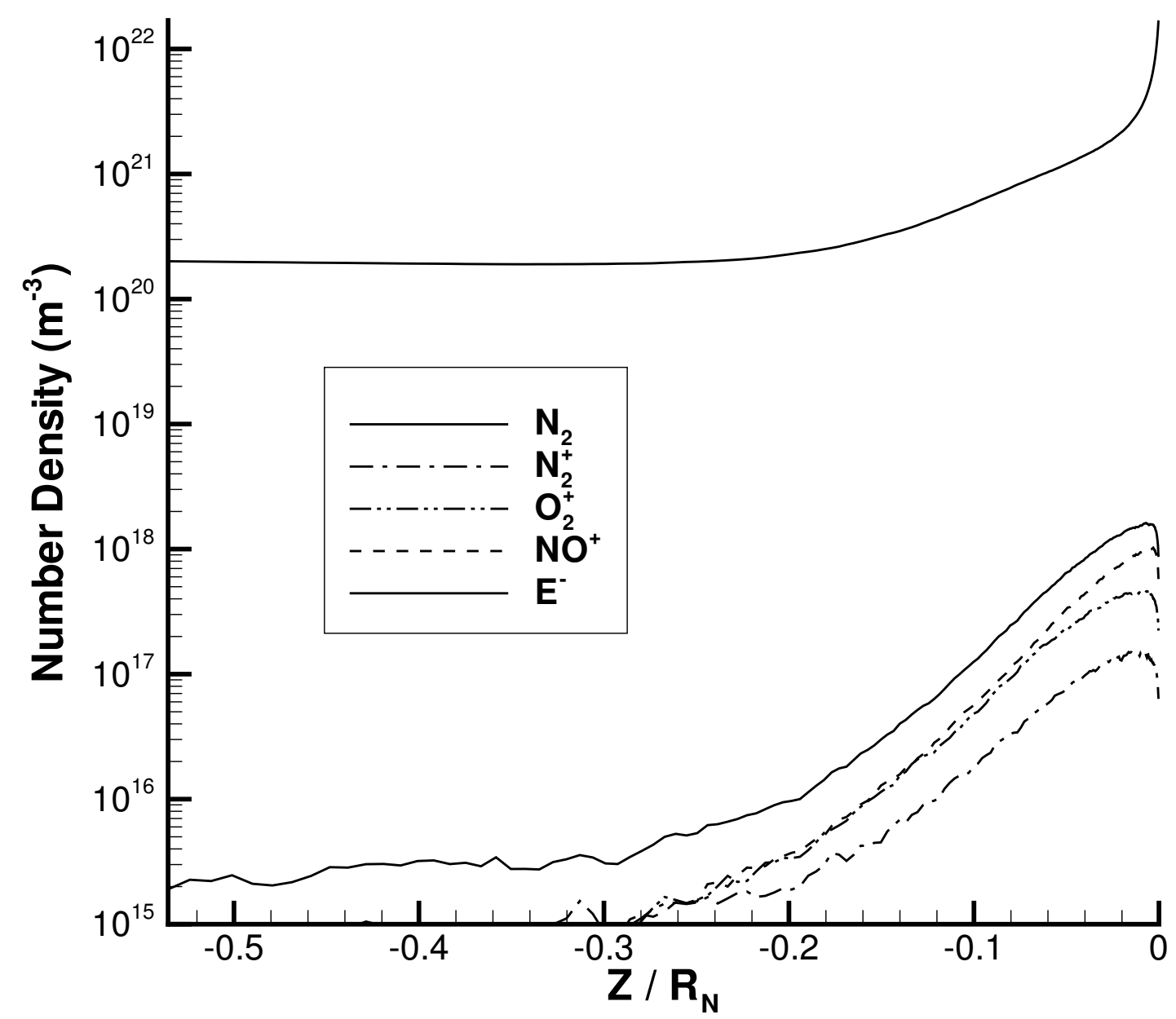

Figure 9. Number density profiles of various species along the stagnation streamline for the RAM-C II nose cap region simulation. 


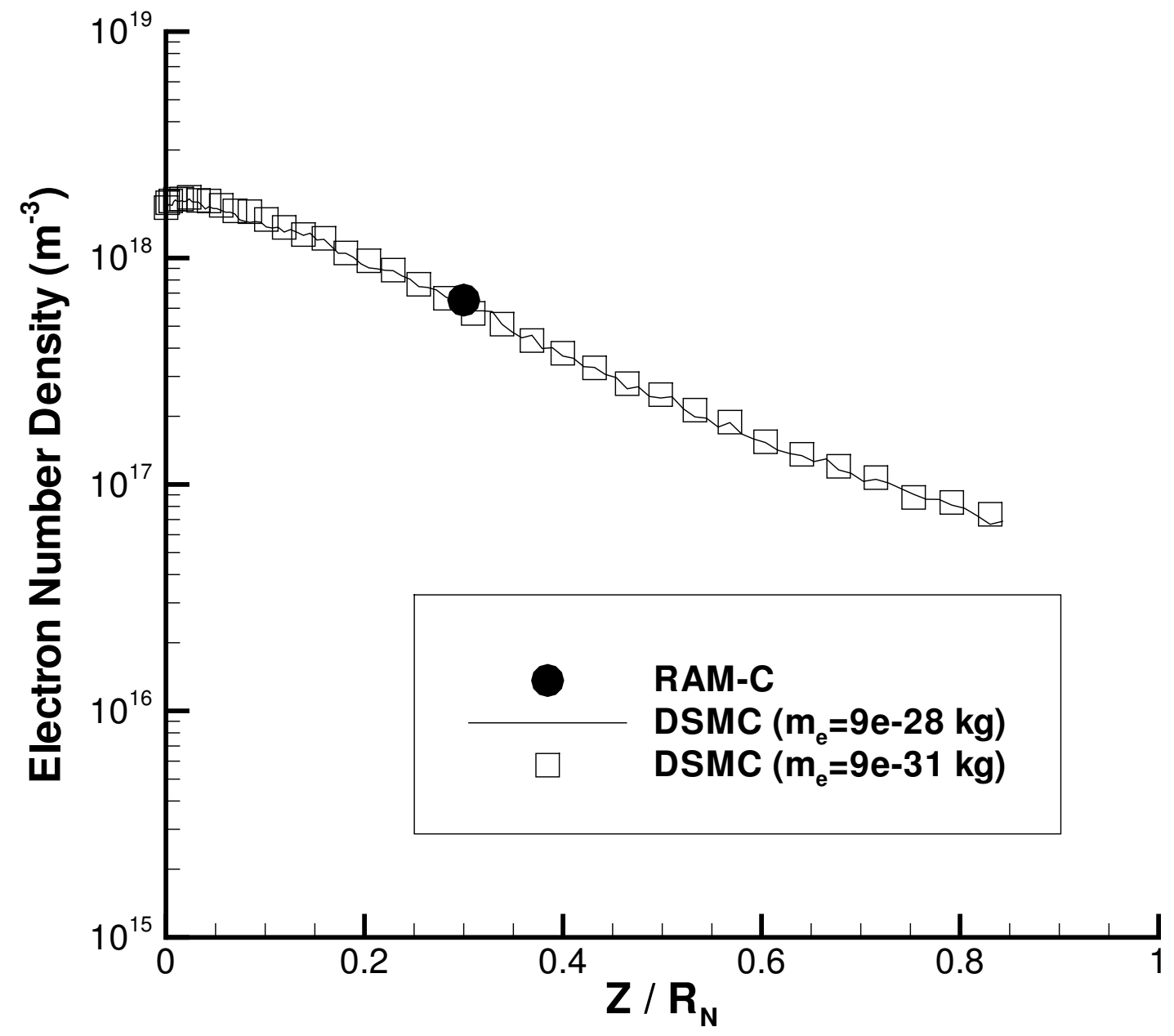

Figure 10. Maximum electron number density as a function of distance along the RAM-C II vehicle. 


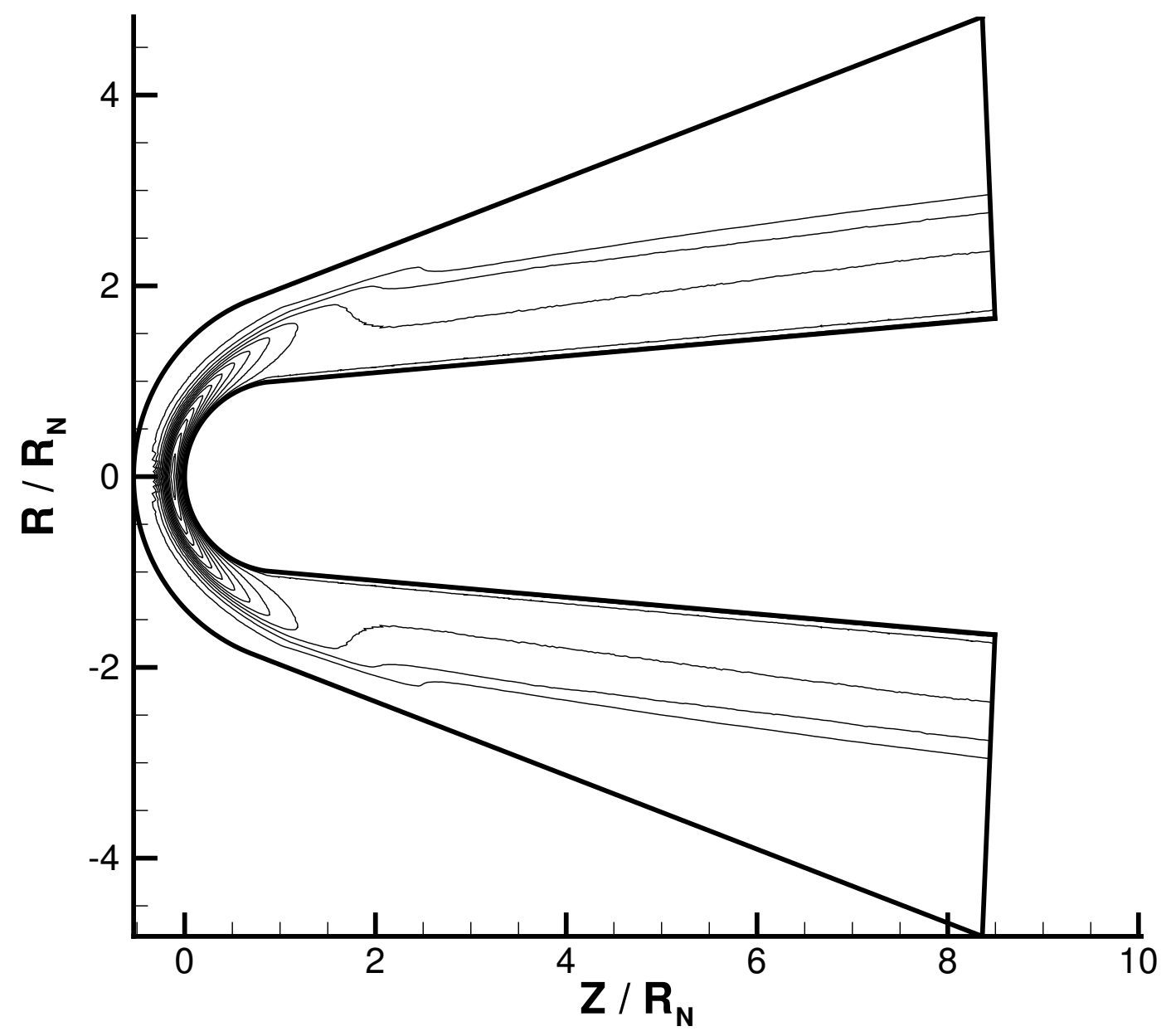

Figure 11. Temperature contours (K) for the full-scale RAM-C II simulation. 


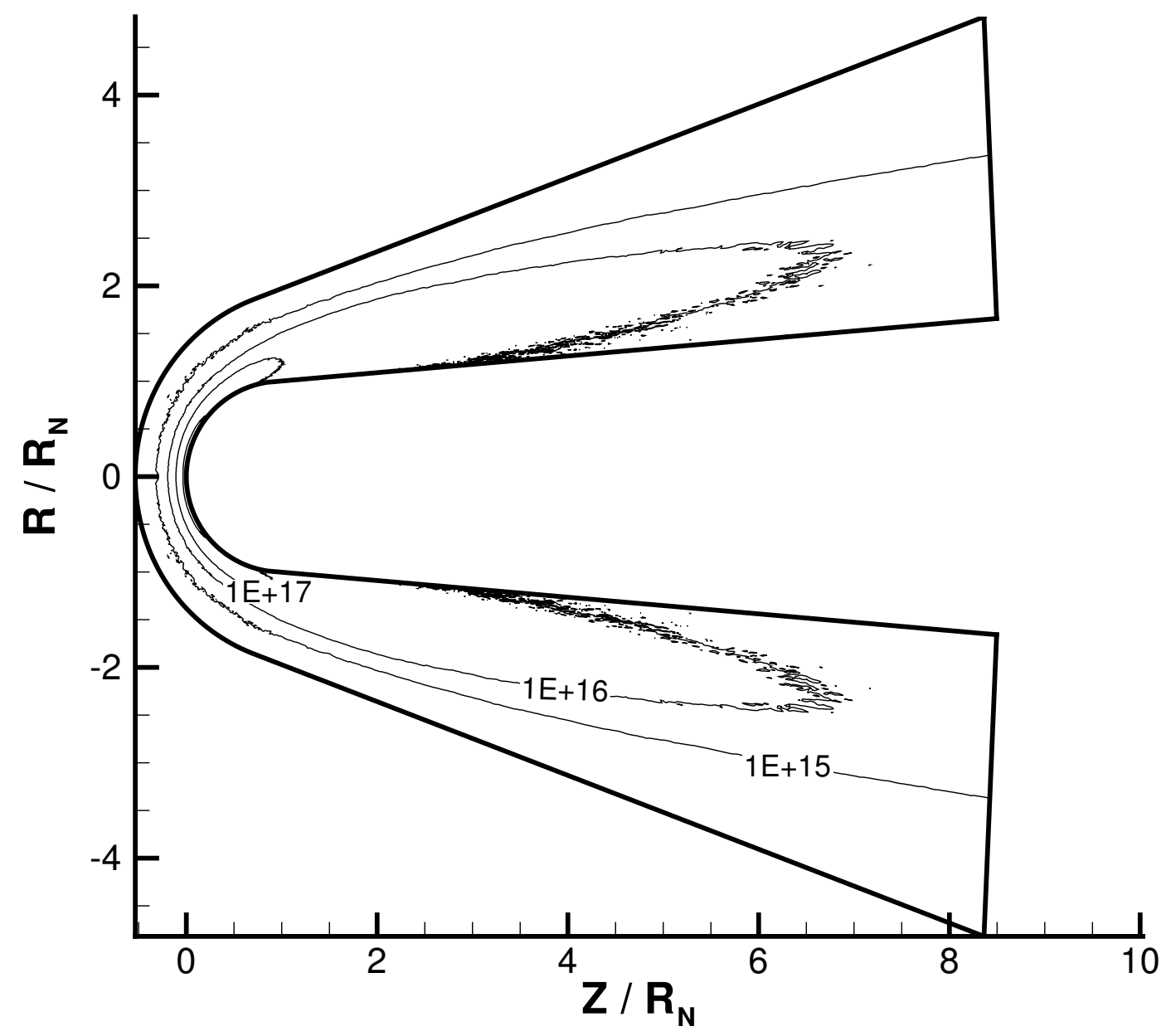

Figure 12. Electron number density contours $\left(\mathrm{m}^{-3}\right)$ for the full-scale RAM-C II simulation. 


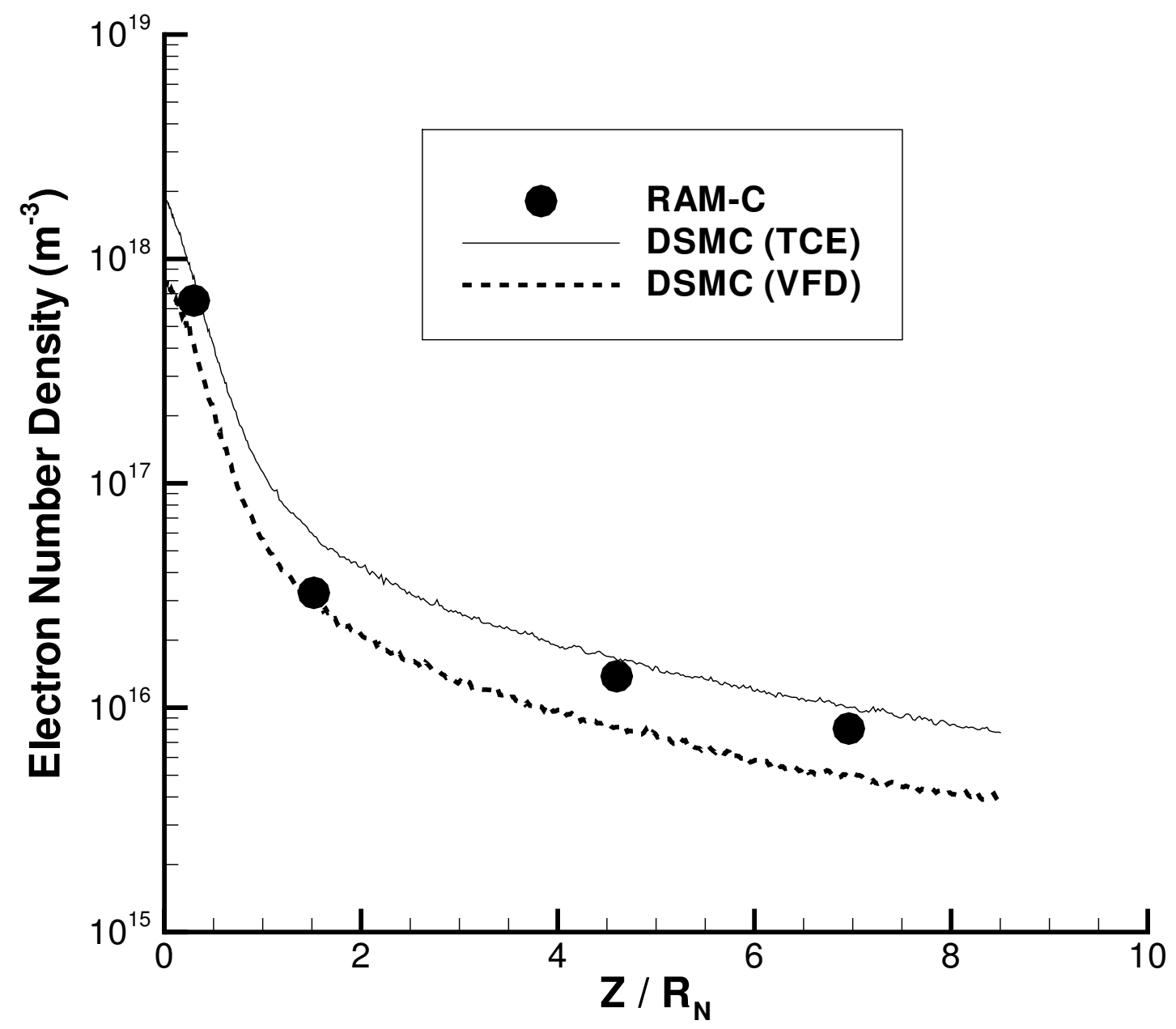

Figure 13. Maximum electron number density as a function of distance along the RAM-C II vehicle. 


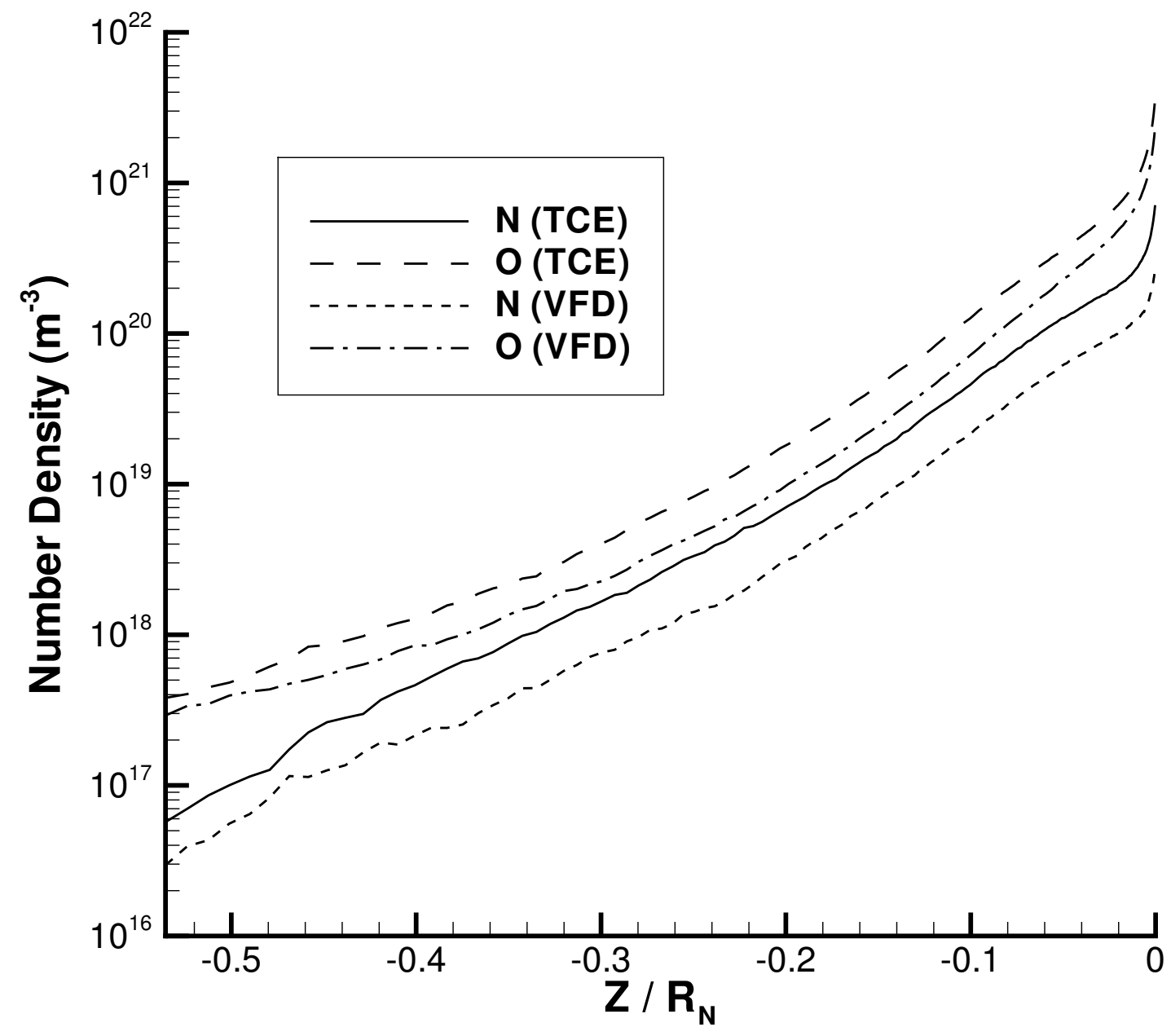

Figure 14. Atom number density along the stagnation streamline. 


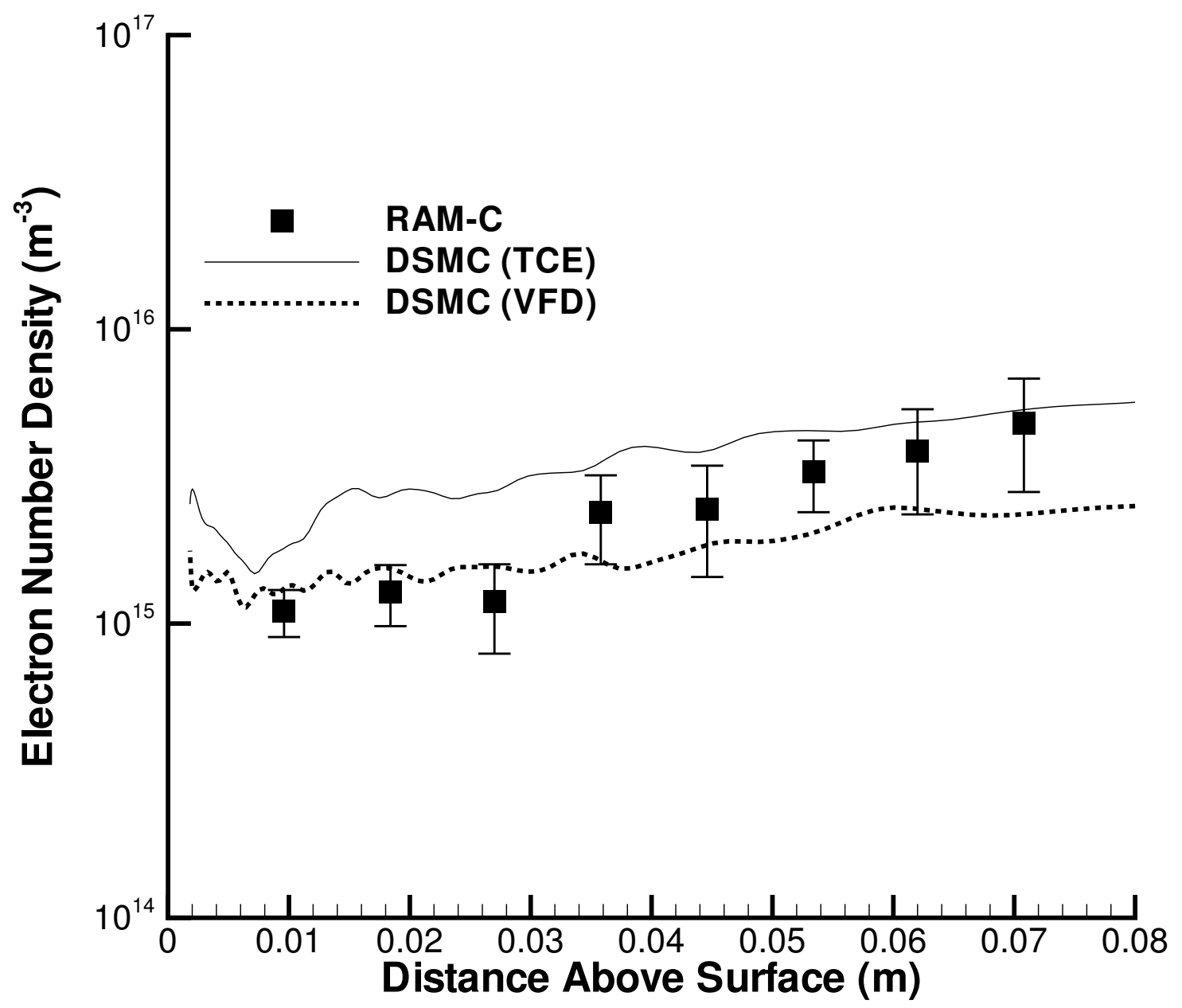

Figure 15. Electron number density as a function of distance from the RAM-C II vehicle at the Langmuir probe location. 